\title{
Unconventional properties of superconducting cuprates
}

\author{
Andrey V. Chubukov ${ }^{1}$ and Dirk K. Morr ${ }^{2}$ \\ ${ }^{1}$ Department of Physics, University of Wisconsin, Madison, WI 53706 \\ 2 University of Illinois at Urbana-Champaign, Loomis Laboratory of Physics, 1100 West Green Street, Urbana, Illinois 61801
}

(October 26, 2018)

\begin{abstract}
We present an explanation of the unusual peak/dip/hump features observed in photoemission experiments on Bi2212 at $T \ll T_{c}$. We argue that these features arise from the interaction of the fermionic quasi-particles with overdamped spin fluctuations. We show that the strong spin-fermion interaction combined with the feedback effect on the spin damping due to superconductivity yields a Fermi-liquid form of the fermionic spectral function for $\omega<2 \Delta$ where $\Delta$ is the maximum value of the superconducting gap, and a non-Fermi-liquid form for $\omega>2 \Delta$. In the Fermi-liquid regime, the spectral function $A\left(\mathbf{k}_{F}, \omega\right)$ displays a quasiparticle peak at $\omega=\Delta$; in the non-Fermi-liquid regime it possesses a broad maximum (hump) at $\omega \gg \Delta$. In between the two regimes, the spectral function has a dip at $\omega \sim 2 \Delta$. We argue that our theory also explains the tunneling data for the superconducting density of states.
\end{abstract}

PACS numbers:71.10.Ca,74.20.Fg,74.25.-q

In recent years, the bulk of studies of cuprate superconductors was focused on their unusual normal state properties. Less attention was paid to the behavior of cuprates in the superconducting state. It was generally believed that the superconducting behavior, even in underdoped cuprates, is rather conventional in the sense that most experiments can be explained in the framework of the BCS-type theory for a $d$-wave superconductor. Recently, however, this belief has been challenged by photoemission experiments on Bi2212 materials [1,2]. These experiments demonstrated that even in slightly overdoped cuprates, the spectral function $A(\mathbf{k}, \omega)$ at $T \ll T_{c}$ and in the momentum region near $(0, \pi)$ where the $d$-wave gap is at maximum is qualitatively different from the one expected for a conventional superconductor. Specifically, in the conventional case, $A(\mathbf{k}, \omega)$ possesses a single sharp peak at $\omega=\sqrt{\Delta_{\mathbf{k}}^{2}+\epsilon_{\mathbf{k}}^{2}}$ where $\Delta_{\mathbf{k}}$ is the superconducting gap and $\epsilon_{\mathbf{k}}$ is the fermionic dispersion. The photoemission data for Bi2212 do show a sharp quasiparticle peak near the Fermi surface, but they also reveal two extra features in $A(\mathbf{k}, \omega)$ : a dip at frequencies right above the peak and a broad maximum (hump) at somewhat larger frequencies. Moreover, as one moves away from the Fermi surface, the sharp peak looses its intensity but does not disperse, while the position of the hump varies with $\mathbf{k}$ and gradually recovers the normal state dispersion.

There have been several phenomenological conjectures in the recent literature that the sharp peak measured in photoemission below $T_{c}$ is related to the one seen in neutron scattering data in $Y B C O$ and is due to the appearance of a dispersionless mode of unknown origin below $T_{c}$ [1,3, [4]. In the present communication, we present an alternative explanation of the photoemission data. We argue that the unusual superconducting properties of cuprates can be explained by a strong interaction between electrons and overdamped spin fluctuations [5]. Specifically, we show that the peak/dip/hump features in the spectral function emerge due to a combination of two effects: (i) an almost complete destruction of the Fermi-liquid behavior which eliminates the quasiparticle peak in the normal state and gives rise to a hump in the spectral function at higher frequencies, and (ii) a reduction of the spin damping at small frequencies in the superconducting state which, as a feedback effect, restores Fermi-liquid behavior of the spectral function in the frequency range $\omega<2 \Delta$. As a result, the spectral function near the Fermi surface possesses a quasiparticle peak at $\omega \sim \Delta$, a dip at $\omega \approx 2 \Delta$ where the spectral function experiences a crossover to a non-Fermi liquid behavior, and a hump at a higher frequency. As $\mathbf{k}$ moves away from the Fermi surface, the hump disperses with $\mathbf{k}$ while the quasiparticle peak only looses its intensity as it cannot move farther in frequency than $2 \Delta$. This behavior fully agrees with the photoemission results [1.2].

We first briefly review the results for the spectral function in the normal state and then discuss our calculations in the superconducting state. One of us has recently shown [6] that the fermionic self-energy due to the spinfermion interaction is almost independent of momentum and has the form $\bar{g}^{2} \Sigma(\omega)=\bar{g}^{2} 2 \omega /\left(1+\sqrt{1-i|\omega| / \omega_{s f}}\right)$ where $\bar{g}^{2} \propto \xi$ is a dimensionless coupling ( $\xi$ is the magnetic correlation length) and $\omega_{s f} \propto\left(\bar{g}^{2} \xi\right)^{-1}$ is the typical relaxation frequency of overdamped spin fluctuations. It was further argued that in cuprates $\bar{g}^{2}$ is large even for overdoped materials so that the selfenergy overshadows the bare frequency dependence in the fermionic propagator, i.e., $G_{n}(\mathbf{k}, \omega) \approx Z /\left(\Sigma(\omega)-\epsilon_{\mathbf{k}}\right)$ where $Z=\bar{g}^{-2}, \epsilon_{\mathbf{k}}=Z \bar{\epsilon}_{\mathbf{k}}$, and $\bar{\epsilon}_{\mathbf{k}}$ is the bare fermionic dispersion. For $\omega<\omega_{s f}, \Sigma(\omega)$ has the Fermi liquid form $\Sigma(\omega) \approx \omega+i \omega|\omega| /\left(4 \omega_{s f}\right)$ and accordingly, the spectral function $A(\mathbf{k}, \omega)$ has a conventional peak at $\omega=\epsilon_{\mathbf{k}}$ though with a reduced residue $Z$. For $\omega>\omega_{s f}$, however, the system crosses over to a region which is in the basin of attraction of the quantum critical point, $\xi=\infty$. In this region, $\bar{g}^{2} \Sigma(\omega) \approx A e^{i \pi / 4}|\omega|^{1 / 2} \operatorname{sgn}(\omega)$ where $A=2 \bar{g}^{2} \omega_{s f}^{1 / 2}$ 


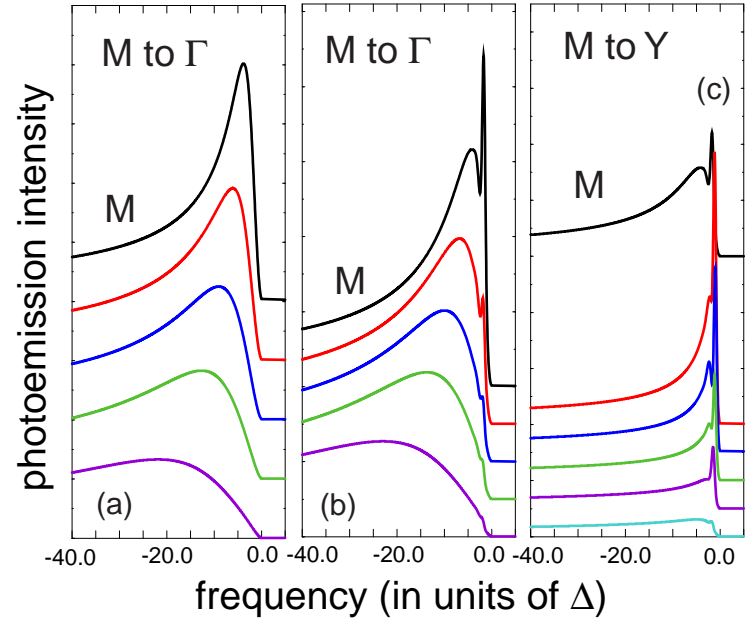

FIG. 1. The calculated quasiparticle spectral function in the normal state $(a)$ and in the superconducting state along $M-\Gamma((0, \pi)-(0,0))$ (b) and $M-Y((0, \pi)-(\pi, \pi))(c)$. The results are presented for $b=5$. For the $M$ point, we used $\bar{\epsilon}=\Delta$.

is independent of $\xi$. As a result, instead of a sharp quasiparticle peak, the spectral function possesses only a broad maximum at $\omega=\epsilon_{\mathbf{k}}^{2} /\left(4 \omega_{s f}\right) \gg \epsilon_{\mathbf{k}}$. Various NMR experiments have shown that $\omega_{s f}$ is anomalously small even if the correlation length is comparable to the interatomic spacing $\left(\omega_{s f} \leq 15 \mathrm{meV}\right.$ even at optimal doping [10]) Since this $\omega_{s f}$ is smaller than the resolution of the photoemission experiments, the experimentally measured $A(\mathbf{k}, \omega)$ only displays a broad maximum even for $\mathbf{k} \approx \mathbf{k}_{F}$ (see Fig. 17a).

Consider now the same system at $T<<T_{c}$. We argue that there are two key effects associated with the superconducting state. First, the quasiparticle Green's function is modified due to the fermionic pairing. In the BCS approximation we have $G_{s c}^{-1}(\mathbf{k}, \omega)=G_{n}^{-1}(\mathbf{k}, \omega)+$ $\left.\Delta_{\mathbf{k}} / Z\right)^{2} G_{n}(-\mathbf{k},-\omega)$ where $\left(\Delta_{\mathbf{k}} / Z\right)^{2}$ is the strength of the $d$-wave pairing susceptibility. In a Fermi-gas $(Z=1)$ $\Delta_{k}$ is the pairing gap. In our case, however, $Z \ll 1$, $G_{n}(\mathbf{k}, \omega)=Z /\left(\Sigma(\omega)-\epsilon_{k}\right)$, and we obtain

$$
G_{s c}(\mathbf{k}, \omega)=Z \frac{\Sigma(\omega)+\epsilon_{\mathbf{k}}}{\Sigma^{2}(\omega)-\left(\Delta_{\mathbf{k}}^{2}+\epsilon_{\mathbf{k}}^{2}\right)} .
$$

For $\omega<\omega_{s f}$, this form of $G_{s c}(\mathbf{k}, \omega)$ again yields a conventional quasiparticle peak at $\omega=\sqrt{\Delta_{\mathbf{k}}^{2}+\epsilon_{\mathbf{k}}^{2}}$. For $\omega>\omega_{s f}$, however, the spectral function takes the form

$$
A(\mathbf{k}, \omega) \propto \sqrt{|\omega|} \frac{|\omega|+E_{k}+\epsilon_{\mathbf{k}} \sqrt{|\omega| /\left(2 \omega_{s f}\right)} \operatorname{sgn}(\omega)}{\omega^{2}+E_{k}^{2}}
$$

where $E_{k}=\left(\Delta_{\mathbf{k}}^{2}+\epsilon_{\mathbf{k}}^{2}\right) /\left(4 \omega_{s f}\right)$, and thus shows the same features as the one in the normal state: it possesses a broad maximum at $\omega=E_{k}$, but no quasiparticle peak.

Clearly, the possibility to observe a quasiparticle peak in cuprates near $\mathbf{k}=(0, \pi)$ where $\Delta_{\mathbf{k}}$ is near its maximum value $\Delta$ depends on the ratio $b=\Delta / \omega_{s f}$. If this ratio is small, then at frequencies comparable to $\Delta$, the system is in the Fermi liquid regime and $A(\mathbf{k}, \omega)$ displays a peak, while if $b \geq 1$, then at $\omega \sim \Delta$, the system is already in the non-Fermi liquid regime where $A(\mathbf{k}, \omega)$ only exhibits a broad maximum. An earlier computation of $b$ in the spin-fluctuation approach [6] has shown that $b$ increases with decreasing doping and becomes larger than one already for slightly overdoped cuprates. At this stage, we therefore only obtain a hump in $A(\mathbf{k}, \omega)$.

However, there is a second effect related to superconductivity which gives rise to a quasiparticle peak in the spectral function even if $b \gg 1$. Indeed, in the above analysis we have so far assumed that $\omega_{s f}$ is independent of frequency. This, however, is not true in the superconducting state as the opening of the superconducting gap reduces the spin damping at low frequencies and hence increases $\omega_{s f}$ in the same frequency range. From this perspective, the parameter $b$ we introduced before is identical to $b_{\infty} \equiv \Delta / \omega_{s f}(\omega=\infty)$ where $\omega_{s f}(\omega=\infty)$ is the value of $\omega_{s f}$ in the normal state, while the existence of a quasiparticle peak is actually determined by $b_{\omega}=\Delta / \omega_{s f}(\omega)$ at $\omega \sim \Delta$.

The spin relaxation frequency $\omega_{s f}$ is inversely proportional to the damping of a spin fluctuation at $\mathbf{Q}=(\pi, \pi)$ due to its decay into a particle-hole pair. To obtain $\omega_{s f}(\omega)$ we hence have to evaluate the imaginary part of the particle-hole bubble. The normal state calculations have been reported previously [6] and yielded a frequency independent $\omega_{s f}=(3 / 16) Z v_{F} \xi^{-1}\left(v_{F}\right.$ is the Fermi velocity). It is essential that this result is independent of $\Sigma$ and is therefore the same as for free fermions. This universality is a general consequence of the fact that the normal state self energy depends only on frequency [8]. Vertex corrections do modify $\omega_{s f}$, but these corrections are very small numerically and can be safely neglected [9.

We now turn to the superconducting state. Here we have to (i) combine two fermionic bubbles made of normal and anomalous Green's functions and (ii) reevaluate the fermionic self-energy $\Sigma(\mathbf{k}, \omega)$ using superconducting Green's functions for intermediate fermions. Simple estimates show that for finite $\Delta, \omega_{s f}$ indeed acquires a frequency dependence and recovers the normal state value only at $\omega \gg \Delta$. Moreover, unlike normal state $G_{n}^{-1}(\mathbf{k}, \omega)$, superconducting $G_{s c}^{-1}(\mathbf{k}, \omega)$ cannot be written as the normal state Fermi-gas result plus a momentumindependent self-energy. As a result, $\omega_{s f}(\omega)$ depends on the self-energy of the intermediate fermions which by itself depends on $\omega_{s f}$. Consequently, the equation for $\omega_{s f}(\omega)$ becomes an integral equation. Below we solve for $\omega_{s f}(\omega)$ assuming that $\Sigma(k, \omega)$ in the superconducting state has the same form as in the normal state albeit with a frequency dependent $\omega_{s f}(\omega)$. We checked by explicitly evaluating the lowest-order self-energy diagram that the terms we omitted by using the normal state rather than the superconducting Green's function change the results by less than $5 \%$ for all frequencies.

To obtain the equation for $b_{\omega}$, we integrated over the 
fermionic momentum in the bubbles and assembling normal and anomalous contributions to the spin damping. This yields

$$
b_{\omega}=\frac{b}{\omega} \operatorname{Re} \int_{0}^{\infty} d \Omega \frac{\Delta^{2}-\Sigma\left(\Omega_{+}\right) \Sigma\left(\Omega_{-}\right)+D\left(\Omega_{+}\right) D\left(\Omega_{-}\right)}{D\left(\Omega_{+}\right) D\left(\Omega_{-}\right)}
$$

where, we remind, $b_{\omega}=\Delta / \omega_{s f}(\omega), b=b_{\infty}=\Delta / \omega_{s f}(\infty)$. Also $\Omega_{ \pm}=\Omega \pm \omega / 2, \Sigma(\Omega)=2 \Omega /\left(1+\sqrt{1-i|\Omega| / \omega_{s f}(\Omega)}\right)$, and $D(\Omega)=\sqrt{\Sigma^{2}(\Omega)-\Delta^{2}}$.

It is instructive to consider the limits of small and large $b$. For $b \ll 1$, the fermionic damping is negligible, $\Sigma(\Omega) \approx \Omega$, and $b_{\omega}$ has the same functional form as the spin damping in the superconducting Fermi gas: it is almost zero for $\omega<2 \Delta$, jumps to $b_{\omega}=b \pi / 2$ at $\omega \geq 2 \Delta$ and gradually approaches $b$ with increasing frequency. A more careful look into Eq.(3) shows that the jump in $b_{\omega}$ at $\omega=2 \Delta$ is a consequence of a singularity in the integrand at $\left|\Omega_{ \pm}\right|=\Delta$. For finite fermionic damping, the singularity is washed out but as long as the damping at $\left|\Omega_{ \pm}\right|=\Delta$ is small (which is clearly the case for small $b), b_{\omega}$ sharply drops below $\omega=2 \Delta$. Consider next what happens when $b \gg 1$. For $\omega \gg \Delta, \omega / \omega_{s f}(\omega) \gg 1$ and hence $\Sigma\left(\Omega_{ \pm}\right) \propto e^{i \pi / 4} \sqrt{\left|\Omega_{ \pm}\right|}$. Using this form of $\Sigma\left(\Omega_{ \pm}\right)$, one can easily verify that the downturn renormalization of $b_{\omega}$ starts already at $\omega \sim \Delta b \gg \Delta$. At smaller frequencies, we expand the integrand in (3) in powers of $\omega$ and obtain $b_{\omega} \sim \omega / \Delta$. For $\omega \sim \Delta$, we then have $b_{\omega} \sim 1$ independently on how large $b$ is. This in turn implies that at these frequencies, the system crosses over into the Fermi liquid regime, and the behavior of $b_{\omega}$ at $\omega \leq \Delta$ is qualitatively the same as for small $b$. Clearly then, $b_{\omega}$ should drop at around $\omega=2 \Delta$ no matter how large $b$ is. Our numerical solution of (3) which we present in Fig. 2 confirms our analytical result. We see that while the high frequency part of $b_{\omega}$ evolves with increasing $b$ from frequency independent to a linear in frequency behavior, a sharp drop in $b_{\omega}$ survives even for large $b$.

The strong reduction of $b_{\omega}$ at low frequencies yields a crossover in the system behavior from a non-Fermi liquid, strong coupling behavior at $\omega>2 \Delta$ to a conventional Fermi-liquid behavior at $\omega<2 \Delta$. In the latter case, the spectral function $A\left(\mathbf{k}_{F}, \omega\right)$ indeed possesses a sharp quasiparticle peak at $\omega=\Delta$ and rapidly decreases at higher frequencies. Combining these two limits, we find three distinct features in $A\left(\mathbf{k}_{F}, \omega\right)$ : a quasiparticle peak at $\omega \sim \Delta$, a dip at $\omega \sim 2 \Delta$ and a hump at $\omega=\Delta b / 4$ which at $b \gg 1$ is parametrically larger than the peak frequency (see Fig. 11). These results are fully consistent with the photoemission data [1]2]. Furthermore, analyzing the form of the spectral function Eq.(1) for various momenta, we find that the quasiparticle peak does not disperse with $\mathbf{k}$ as the region of Fermi liquid behavior does not extend farther than $2 \Delta$ away from the Fermi surface. Instead, the peak gradually decreases in the amplitude as one moves away from $\mathbf{k}_{F}$. This is clearly

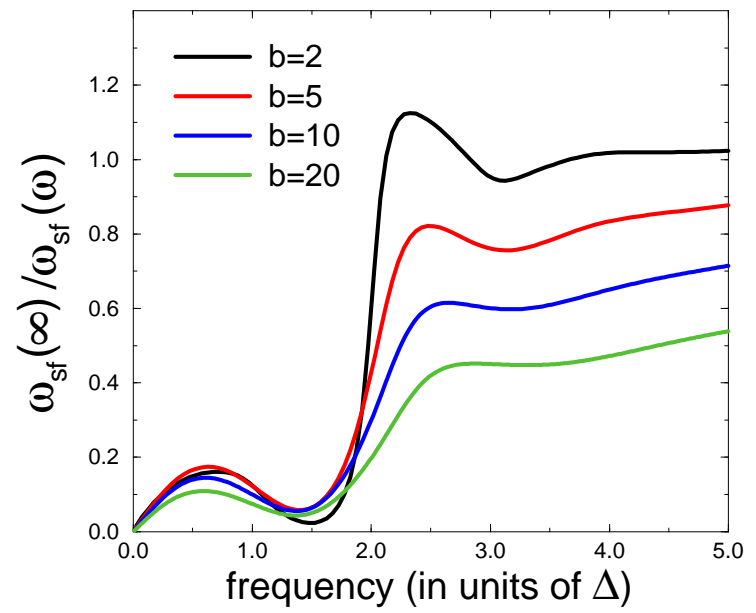

FIG. 2. The solution of the integral equation for the frequency dependent spin-fluctuation frequency $\omega_{s f}(\omega)$ in the superconducting state. In the normal state, $\omega_{s f}=\omega_{s f}(\infty)$. For all $b=\Delta / \omega_{s f}(\infty), \omega_{s f}^{-1}(\omega)$ sharply drops at around $2 \Delta$.

seen in Fig. 1 b b,c. In contrast, the position of the hump follows $\omega \propto\left(\Delta_{k}^{2}+\epsilon_{k}^{2}\right)$. Near $(0, \pi)$, where $\epsilon_{\mathbf{k}}$ is small, the dispersion is weak, whereas further away from the Fermi surface it disperses with $\mathbf{k}$ and gradually recovers the normal state dispersion. In Fig 3, we plotted the frequency position of the quasiparticle peak and hump in the superconducting state vs. the normal state position of the hump. This dependence on momentum is also fully consistent with the photoemission data [1].

Next we compute the density of states $N(\omega)$ in the superconducting state assuming that the dominant contribution to $N(\omega)$ comes from momenta near $(0, \pi)$. Integrating $A(\mathbf{k}, \omega)$ over $\epsilon_{\mathbf{k}}$, we obtain

$$
N(\omega) \propto \operatorname{Re} \frac{\Sigma(\omega)}{\sqrt{\Sigma^{2}(\omega)-\Delta^{2}}} .
$$

The plots of $N(\omega)$ for various $b$ are presented in Fig. 4 . We see that for all values of $b, \mathrm{~N}(\omega)$ possesses a peak at $\omega=a \Delta$ where $a \approx 1$ for small $b$ and gradually increases with increasing $b$. For larger $b, N(\omega)$ displays a dip at frequencies slightly larger than $2 \Delta$; the amplitude of the dip increases with $b$. Above the dip, $N(\omega)$ increases as $\sqrt{\omega}$ and eventually saturates. These results are in full agreement with the tunneling data in Ref. [7] except for the experimentally observed anisotropy between $N(\omega)$ for positive and negative frequencies for which we do not have an explanation.

Furthermore, we find that for large $b$ (i.e., for underdoped cuprates), the region near $(0, \pi)$ yields a substantial contribution to $N(\omega)$ even for $\omega<\Delta$, which possibly overshadows the contribution from the nodes of $\Delta_{\mathbf{k}}$. Specifically, we found from Eq.(19) that $N(\omega) \propto \omega^{3}$ for very small frequencies which crosses over to $N(\omega) \propto \omega$ around $\omega \sim \Delta / 2$. This behavior is very similar to the one observed in underdoped cuprates. On the other hand, for 


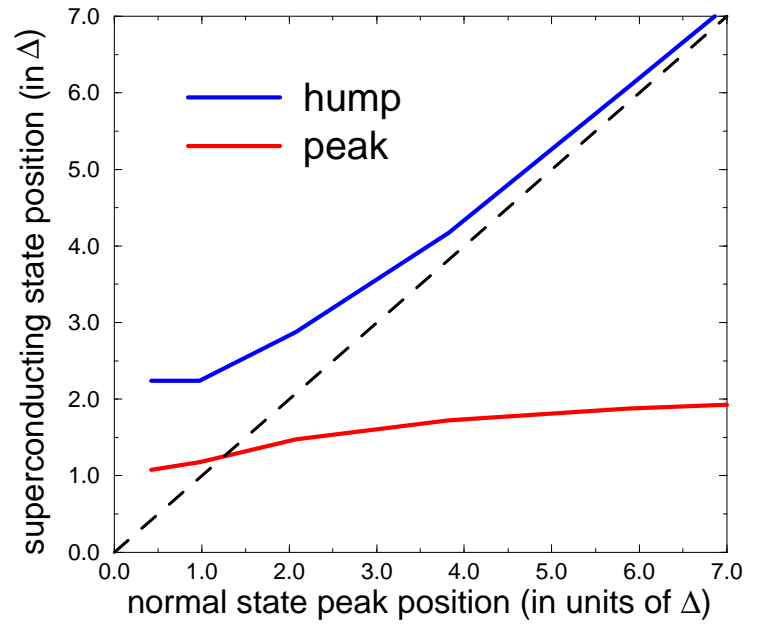

FIG. 3. The frequency position of the quasiparticle peak and the hump in the superconducting state versus position of the hump in the normal state obtained from Fig. 1.

small $b$ (i.e., for strongly overdoped cuprates), the contribution from the nodes is indeed relevant and our results underestimate $N(\omega)$ for $|\omega| \leq \Delta$.

Finally, we discuss the value of the gap and its variation with doping. Consider first the location of the quasiparticle peak in $A(\mathbf{k}, \omega)$. Our theory predicts that at some distance away from the Fermi surface, it should be located at $\omega \approx 2 \Delta$. Applying this result to nearly optimally doped Bi2212 materials studied in Ref. [1.2], we obtain $\Delta \sim 25-30 \mathrm{meV}$. Almost the same result is obtained by extracting $2 \Delta$ from the onset of the dip in measured $N(\omega)$ [7]. Furthermore, for $T_{c}=83 K$ underdoped material, our analysis of the tunneling data yields almost the same value of $\Delta$ as at optimal doping. We therefore argue that $\Delta$ almost saturates around optimal doping, and the observed increase of the peak frequency in $N(\omega)$ with decreasing doping is mostly due to strong coupling effects which shift the peaks towards higher frequencies. Notice also that a comparison of $\Delta$ with $\omega_{s f}(\infty)$ extracted from NMR data 10] in the normal state confirms our assertion that $b>1$ already at optimal doping.

To summarize, in this paper we present the explanation of the unusual peak/dip/hump features observed in photoemission experiments on Bi2212 at $T \ll T_{c}$. We argue that these features are explained by the interaction of fermionic quasiparticles with overdamped spin fluctuations. We show that the strong spin-fermion interaction combined with the feedback effect on the spin damping due to fermionic pairing yield a Fermi-liquid form of the fermionic spectral function at $\omega<2 \Delta$ where $\Delta$ is the maximum value of the superconducting gap, and a non-Fermi-liquid form for $\omega>2 \Delta$. In the Fermiliquid regime, the spectral function near $\mathbf{k}_{F}$ displays the quasiparticle peak at $\omega \approx \Delta$; in the non-Fermi-liquid regime above $2 \Delta$ it possesses a broad maximum (hump) at $\omega \gg \Delta$. In between the two regimes, the spectral func-

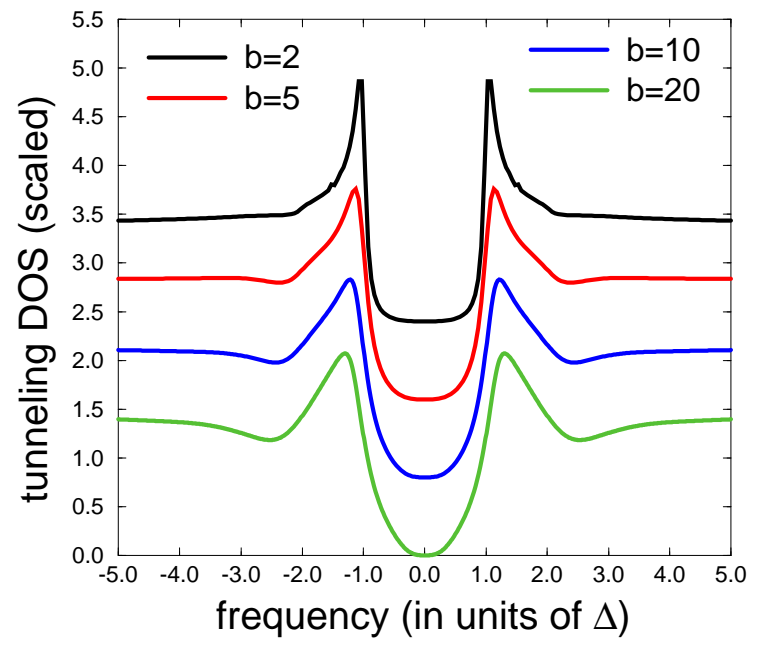

FIG. 4. The tunneling density of states in the superconducting state. Observe the development of a dip with increasing $b$.

tion has a dip at $\omega \sim 2 \Delta$. We argue that our theory also explains the tunneling data for the superconducting density of states. We predict that the superconducting gap saturates around optimal doping, and that the observed increase of the peak frequency in the tunneling density of states with decreasing doping is chiefly due to strong coupling effects.

It is our pleasure to thank G. Blumberg, R. Joynt, M. Norman, D. Pines and J. Schmalian for useful conversations. The research was supported by NSF DMR9629839 (A.C.) and in part by STCS through NSF DMR9120000 (D.M.).

[1] M. R. Norman et al., Phys. Rev. Lett. 79, 3506 (1997).

[2] Z-X. Shen et al, Science 280, 259 (1998).

[3] G. Blumberg et al., Science 278, 1427 (1997).

[4] S.C. Zhang, Science 285, 1089 (1997)

[5] A.P. Kampf and J.R. Schrieffer, Phys. Rev. B 42, 7967 (1990); P. Monthoux and D. Pines, Phys. Rev. B 47, 6069 (1993); D.J. Scalapino, Phys. Rep. 250, 329 (1995).

[6] A. Chubukov, Europhys. Lett, submitted; A.V. Chubukov and J. Schmalian, Phys. Rev B 57, R11085 (1998); see also A.J. Millis, ibid 45, 13047 (1992); S. Sachdev and J. Ye, Phys. Rev. Lett. 70, 3339 (1993).

[7] Ch. Renner et al., Phys. Rev. Lett. 80, 149 (1998).

[8] L. Kadanoff, Phys. Rev. 132, 2073 (1963).

[9] A. Chubukov and D. Morr, Phys. Rep., 288, 355 (1997).

[10] V. Barzykin and D. Pines, Phys. Rev. B, 52, 13585 (1995).

[11] J.R. Schrieffer, Theory of Superconductivity, Frontiers in Physics, Addison Wesley, (1988). 